\title{
The clinical relevance of pathogen Aerococcus urinae identification by MALDI-TOF mass spectrometry: a case of sepsis in 86-year-old Caucasian male
}

\author{
Jari Intra, ${ }^{1}$ Cecilia Sarto, ${ }^{2}$ Giuseppe Serra, ${ }^{3}$ Paolo Brambilla ${ }^{2}$ \\ ${ }^{1}$ Clinical Chemistry Laboratory, University of Milano-Bicocca, Azienda Socio Sanitaria Territoriale di Monza ASST- \\ Monza, St Gerardo Hospital, Monza; ${ }^{2}$ Department of Laboratory Medicine, University of Milano-Bicocca, Azienda \\ Socio Sanitaria Territoriale di Monza ASST-Monza, Desio Hospital, Desio; ${ }^{3}$ Department of Internal Medicine, Azienda \\ Socio Sanitaria Territoriale di Monza ASST-Monza, Desio Hospital, Desio, Italy
}

\begin{abstract}
Summary
The infrequency of urinary tract and blood stream infections caused by Aerococcus urinae is most probably due to the difficulties in the identification of this bacterium using standard microbiological methods. With the introduction of more sensitive and accurate techniques in clinical microbiology, such as genetic approaches and Matrix-Assisted Laser Desorption/Ionization-Time Of Flight (MALDI-TOF) mass
\end{abstract}

Correspondence: Jari Intra, Clinical Chemistry Laboratory, University of Milano-Bicocca, Azienda Socio Sanitaria Territoriale di Monza ASST-Monza, St Gerardo Hospital, via Pergolesi 33, 20900, Monza (MB), Italy.

Tel.: +390392336903 - Fax: +390392333011

E-mail: j.intra@asst-monza.it

Key words: Aerococcus urinae, sepsis, MALDI-TOF, urinary tract infection, identification.

Acknowledgments: We gratefully acknowledge the technical support of laboratory personnel of Desio Hospital, and Dr. Natalia Tiberti and Dr. Elena Intra for reviewing the manuscript.

Contributions: The authors contributed equally.

Conflict of interest: The authors declared no conflict of interest.

Availability of data and materials: All data are available within the text.

Informed consent: Informed consent to publish was obtained from the individual included in the study.

Received for publication: 17 June 2020.

Accepted for publication: 23 July 2020

${ }^{\circ}$ Copyright: the Author(s), 2020

Licensee PAGEPress, Italy

Microbiologia Medica 2020; 35:8965

doi:10.4081/mm.2020.8965

This article is distributed under the terms of the Creative Commons Attribution Noncommercial License (by-nc 4.0) which permits any noncommercial use, distribution, and reproduction in any medium, provided the original author(s) and source are credited. spectrometry (MS), the incidence of infections due to A. urinae increased. Herein, we described a case of urinary tract and bloodstream infection caused by $A$. urinae, which occurred in an 86-year-old Caucasian man with a previous history of prostate cancer. The identification of $A$. urinae was performed by MALDI-TOF MS, since this microorganism cannot be identified by biochemical reactions. In this report, we highlight the need to consider MALDI-TOF MS as technique of choice for A. urinae identification in the presence of subjects with predisposing factors, such as old age, male gender, and genitourinary tract pathologies.

\section{Introduction}

Aerococcus urinae is a Gram-positive, catalase-negative, alpha-hemolytic coccus growing in small clusters belonging to genus Aerococcus that presents a ubiquitous distribution in the environment, such as in soil, air and normal microbiota of different mammals (1-3). In 1992, A. urinae was recognized as a separate species from Aerococcus viridans with the use of $16 \mathrm{~S}$ ribosomal subunit sequencing, and now, with improvements in microorganism identification methods, seven Aerococcus species have been identified $(1,3)$. A urinae was reported to be a rare cause of human urinary tract infections (estimated prevalence rate of less than $1 \%$ ) and a rare cause of bacteremia (less than 3 cases per 1 million inhabitants per year) $(1,3)$. Risk factors include elderly male subjects and comorbidities associated to genitourinary tract $(1,3)$. The incidence of human infections caused by this pathogen is however underestimated since the identification of aerococci often fails due to the colony morphology and microscopic appearance in Gram-stain similar to staphylococci, and to the antibiotic resistance patterns similar to those of enterococci $(1,3,4)$. Moreover, a variety of common diagnostic systems based on biochemical reactions do not recognize aerococci, thus increasing the misidentification in many laboratories world-wide $(1,5)$. However, the introduction of MALDI-TOF MS and genetic techniques as $16 \mathrm{~S}$ ribosomal subunit sequencing for bacteria identification in diagnostic microbiological laboratories has increased the frequency of detection of infections caused by $A$. urinae in human $(1,3,4,6-9)$.

Here, we present a case of $A$. urinae urosepsis in an 86-yearold Caucasian man with the intent to highlight the clinical importance of using MALDI-TOF for aerococci identification since 
common microbiological approaches miss to identify these microorganisms.

\section{Case Report}

On 24 November 2019, an 86-year-old Caucasian man was admitted to the hospital with altered consciousness and hematuria. Following hospitalization, the patient developed dyspnea, fever and emesis. Medical history included hypertension, peripheral vascular disease, depression, severe cognitive impairment and, in 2006, diagnosis of prostate cancer (Gleason grade $4+3$ ) treated with radiotherapy ( $74 \mathrm{~Gy}$ in 37 fractions).

Laboratory findings were as follows: red blood cells (RBC) $5.13 \times 10^{6} / \mu \mathrm{L}$, hemoglobin $(\mathrm{Hb}) 15.7 \mathrm{~g} / \mathrm{dL}$, haematocrit (Hct) $46.6 \%$, peripheral white blood cells (WBC) count $5,800 / \mu \mathrm{L}$, platelet count (PLT) $248,000 / \mu \mathrm{L}, \mathrm{C}$-reactive protein (CRP) 19.63 $\mathrm{mg} / \mathrm{L}$ (reference range: $0.0-5.0 \mathrm{mg} / \mathrm{L}$ ), procalcitonin $0.1 \mathrm{ng} / \mathrm{mL}$ (reference range: $<0.5 \mathrm{ng} / \mathrm{mL}$ ), and creatinine $1.84 \mathrm{mg} / \mathrm{dL}$ (reference range: $0.7-1.2 \mathrm{mg} / \mathrm{dL}$ ). An ECG was normal. Urinalysis showed WBC too numerous to count, 5-10 RBC per optical field, many bacteria, and positive test results of blood haemoglobin, leukocyte esterase, and protein. Blood and urine cultures were performed, and intravenous ceftriaxone administration was started ( $2 \mathrm{~g} /$ day $)$ on the presumptive diagnosis of urinary tract infection. One day after, the laboratory values showed RBC $4.56 \mathrm{x}$ $10^{6} / \mu \mathrm{L}, \mathrm{Hb} 14.1 \mathrm{~g} / \mathrm{dL}$, Hct $40.1 \%$, PLT $166,000 / \mu \mathrm{L}$, and elevated results of WBC $18,500 / \mu \mathrm{L}$ with $17.76 \times 10^{3} / \mu \mathrm{L}$ neutrophils $(96$ $\%$, CRP $131.47 \mathrm{mg} / \mathrm{L}$, procalcitonin $183.28 \mathrm{ng} / \mathrm{mL}$, and creatinine $2.34 \mathrm{mg} / \mathrm{dL}$. An echotomography showed a small size right kidney, and no hydronephrosis, and a chest X-.ray excluded pulmonary thickening

The urine cultures resulted positive, although the microorganism was not identified by Vitek 2 system (bioMérieux, France). Grown bacteria were therefore analyzed using Vitek ${ }^{\circledR}$ MALDI-TOF MS system (bioMérieux, France), and Aerococcus urinae was identified with $99.9 \%$ confidence. Vitek ${ }^{\circledR} 2$ GP-AST (Antimicrobial Susceptibility Testing) cards were used to achieve a rapid and accurate detection of susceptibility and resistance. According to EUCAST 2020 clinical breakpoints (10), the isolate was susceptible to ampicillin (Minimum inhibitory concentration (MIC): $0.047 \mathrm{mg} / \mathrm{L}$ ), clindamycin (MIC: $0.38 \mathrm{mg} / \mathrm{L}$ ), penicillin (MIC: $0.047 \mathrm{mg} / \mathrm{L}$ ), ciprofloxacin (MIC: $1 \mathrm{mg} / \mathrm{L}$ ), and vancomycin (MIC: $0.75 \mathrm{mg} / \mathrm{L}$ ), while no resistance was detected. The blood cultures were also positive, revealing Gram-positive cocci forming pairs, tetrads and clusters resembling staphylococci on gram staining. Subcultures on blood agar plates at $37^{\circ} \mathrm{C}$ in $5 \% \mathrm{CO}_{2}$ were performed. Based on the findings on urine cultures, grown bacteria were only identified by Vitek ${ }^{\circledR}$ MALDI-TOF MS system, and Aerococcus urinae was identified with a score of $99.9 \%$. The isolate presented the same antimicrobial patterns of strains isolated from urine culture. Following the preliminary evidence of Gram-positive bacteria resembling staphylococci, and due to an initial worsening of respiratory mechanics and a higher CRP value (from 131.47 to $197.25 \mathrm{mg} / \mathrm{L}$ ), antibiotic regimen was changed to Cefepime ( $1 \mathrm{~g}$ twice a day) since $26^{\text {th }}$ November.

After thirteen days of aforesaid antimicrobial treatment, laboratory results were as follows: RBC $4.06 \times 10^{6} / \mu \mathrm{L}, \mathrm{Hb} 12.5$ $\mathrm{g} / \mathrm{dL}$, Hct $37.5 \%$, PLT $180,000 / \mu \mathrm{L}$, WBC $6.600 / \mu \mathrm{L}$ with $4.96 \mathrm{x}$ $10^{3} / \mu \mathrm{L}$ neutrophils $(71 \%)$, CRP $38.01 \mathrm{mg} / \mathrm{L}$, procalcitonin 0.26 $\mathrm{ng} / \mathrm{mL}$, and creatinine $1.99 \mathrm{mg} / \mathrm{dL}$. Follow-up negative urine and blood cultures confirmed the success of therapy.

\section{Discussion and Conclusions}

Aerococcus urinae is a microorganism that was isolated in 1953 in lobsters, and considered of no clinical significance. In 1967, the first case of $A$. urinae infection in human was described (6). A. urinae is a Gram-positive, catalase-negative coccus growing in tetrads or clusters. However, A. urinae isolates may easily be identified as viridans streptococci or staphylococci on gram staining due to their morphological similarities. The difficult determination of a correct species most probably contributes in underestimating the incidence of infections due to this microorganism. Currently, A. urinae is responsible of 0.15 to $0.54 \%$ of urinary tract infections, and it is cause of 0.03 to $0.05 \%$ of invasive infections, such as bloodstream, peritonitis, and vertebral osteomyelitis $(1,6)$. In addition, there are numerous case reports of aerococcal endocarditis infections, particularly caused by $A$. urinae $(1,6)$. Reviewing the literature, advanced age (i.e. $>65$ years), male gender, urologic pathologies, and systemic conditions, such as prostate hyperplasia, diabetes mellitus and dementia, are the best-known risk factors for A. urinae infections (1, 6 ), several of which were presented by our patient (old age, male gender, and treated prostatic cancer). The genitourinary tract seems to be the potential entry of this organism, since aerococci are part of the normal flora of urinary tract, as previously reported in numerous cases $(2,3,6,11)$. In agreement with these reports, we isolated $A$. urinae from both urine and blood samples. However, the diagnosis of $A$. urinae infection is severely limited by the difficulties to identify this microorganism. Gram-staining does not allow species determination, and biochemical reactions, such as leucine-aminopeptidase, hyppurate-hydrolysis and pyrrolidinyl-aminopeptidase, are laborious and time-consuming tests, poor to identify aerococcus species $(1,11)$. Although genetic approaches like 16S rRNA sequencing are the gold standard for aerococci identification, they are expensive and require trained personnel $(1,11)$. The introduction of MALDI-TOF MS in routine laboratories as the primary technique for species determination overcame most of the limits of aerococci identification $(1,4,6$ 9,12 ). The aim of this case report is to highlight the clinical importance of MALDI-TOF MS in aerococcus identification, thus revealing the true incidence of this microorganism. Currently, no guidelines recommending the use of a specific method as primary approach in aerococci identification exist. We suggest that in subjects presenting the risk factors, species determination should be performed by MALDI-TOF MS method, if genetic approaches are not available. In addition to the obvious clinical impact for patients, the correct identification of $A$. urinae might also permit to perform accurate studies on antibiotic sensitivities of this species $(1,6)$. Reviewing in vitro studies, variable resistance to clindamycin, erythromycin, and levofloxacin, and sensitivity to amoxicillin, cefotaxime, ceftriaxone, doxycycline, linezolid, meropenem, penicillin, rifampin, and vancomycin were observed $(1,4,6)$. In our case, $A$. urinae isolates are completely sensitive to all antibiotic tested.

In conclusion, the results of the present Case Report is of relevant importance as it reinforces what is reported in previous studies. Given that there is a tendency to misidentify aerococci microorganisms, the use of MALDI-TOF is of high clinical importance, especially in the presence of subjects with predisposing factors (old age, male gender, and genitourinary tract pathologies).

\section{References}

1. Rasmussen M. Aerococcus: an increasingly acknowledged human pathogen. Clin Microbiol Infect 2016;22:22-7. 
2. Meletis G, Chatzidimitriou D, Tsingerlioti F, et al. An initially unidentified case of urinary tract infection due to Aerococcus urinae. New Microbiol 2017;40:221-2.

3. Yabes JM, Perdikis S, Graham DB, Markelz A. A rare case of Aerococcus urinae infective endocarditis in an atypically young male: case report and review of the literature. BMC Infect Dis 2018;18:522.

4. Carkaci D, Nielsen XC, Fuursted K, et al. Aerococcus urinae and Aerococcus sanguinicola: Susceptibility Testing of 120 Isolates to Six Antimicrobial Agents Using Disk Diffusion (EUCAST), Etest, and Broth Microdilution Techniques. Open Microbiol J 2017;11:160-6.

5. Cattoir V, Kobal A, Legrand P. Aerococcus urinae and Aerococcus sanguinicola, two frequently misidentified uropathogens. Scand J Infect Dis 2010;42:775-80.

6. Figueroa Rodriguez F, Faieta Lasarcina A, Davila Grijalva F. Mitral. Valve Endocarditis with Perforation from a Urinary Source: An Unusual Case and Literature Review. Case Rep Cardiol 2019;2019:5496851.

7. Christensen JJ, Dargis R, Hammer M, et al. Matrix-assisted laser desorption ionization-time of flight mass spectrometry analysis of Gram-positive, catalase-negative cocci not belonging to the Streptococcus or Enterococcus genus and benefits of database extension. J Clin Microbiol 2012;50:1787-91.

8. Senneby E, Nilson B, Petersson AC, Rasmussen M. Matrixassisted laser desorption ionization-time of flight mass spectrometry is a sensitive and specific method for identification of aerococci. J Clin Microbiol 2013;51:1303-4.

9. Senneby E. Comment on: A Case of Aerococcus urinae, Vertebral Osteomyelitis. J Glob Infect Dis 2016;8:160.

10. The European Committee on Antimicrobial Susceptibility Testing. Breakpoint tables for interpretation of MICs and zone diameters. Version 10.0, 2020. http://www.eucast.org.

11. Lee MY, Kim MH, Lee WI, et al. A Case of Sepsis in a 92Year-Old Korean Woman Caused by Aerococcus urinae and Identified by Sequencing the 16S Ribosomal RNA Gene. Lab Med 2016;47:e15-7.

12. de Jong MF, Soetekouw R, ten Kate RW, Veenendaal D. Aerococcus urinae: severe and fatal bloodstream infections and endocarditis. J Clin Microbiol 2010;48:3445-7. 